\title{
EGFR Amplification Analysis Was Performed
}

National Cancer Institute

\section{Source}

National Cancer Institute. EGFR Amplification Analysis Was Performed. NCI Thesaurus.

Code C160353.

An indication that EGFR amplification analysis was performed during the study. 